\title{
CINCO TEXTOS ORACULARES MAYAS
}

\author{
rené ACUNAA \\ Centro de Estudios Mayas \\ David Bolles
}

\section{Auguralia dicta}

Entre los escritos compuestos por los mayas a principios de la época colonial, hay una serie de augurios denominados $U$ tzol than ah kinob, "interpretaciones de los augures", que la tradición literaria antigua y moderna está de acuerdo en llamar "profecías mayas". Son un conjunto de oráculos, de desigual longitud, atribuidos a los presuntos profetas Ah Kauil Chhel, Ah Na Hau Pech, Ah Na Puc Tun, Ah Xupan Nauat, Chilam Balam y Patzin Yaxum Chan. Cinco de ellos, según se dice, intervinieron en una junta sacerdotal celebrada en Tancah Mayapán "in the time of the Zuya people, in the time of Itza" (Roys, 1967: 164). Dato que, al situar en estricta contemporaneidad a los cinco profetas, resulta conflictivo. ${ }^{1}$

Algunos de estos oráculos hablan del arribo de hombres blancos o extraños, portadores de una religión nueva. Por esta razón, tan conveniente a la aspiración que tenían los misioneros de legitimar su mensaje evangélico en Yucatán, estas piezas tempranas de la literatura maya colonial son las que han recibido, desde que los propios frailes las difundieron, la mayor atención de los estudiosos foráneos. Lizana (1633),

${ }^{1}$ No tengo noticia de que se haya hecho ya un escrutinio sistemático de los Chilames para el efecto de contrastar y evaluar la información biográfica que contienen relativa a los presuntos profetas mayas. Sobre este tema, el interesado hará bien en leer el Apéndice D al Chilam Balam de Chumayel (Roys, 1967: 182-187) y, desde luego, los comentarios y notas que dicho autor puso a su traducción de las profecías. Recomendables son, asimismo, los trabajos de éste, contenidos en el volumen 10, números 48 49 y 51, de Contributions to American Anthropology and History, Pub. 585 (Washington, D.C., 1949). 
el primero en darles el nombre de "profecías", entregó a la estampa cinco de ellas, omitiendo la atribuida a Ah Xupan Nauat. Su fuente, un presumible manuscrito antiguo existente en Oxkutzcab que recoge el Códice Pérez (en adelante, CP, ms., pp. 166-170), también la omitía. Más tarde, López de Cogolludo (1957, lib. 2, cap. xI: 97-100), en la tradición de su predecesor, y Villagutierre de Soto-Mayor (1701: 36-37) ofrecieron la versión española de dichos textos. Su orden, y en algunos casos la traducción, están alterados. A partir de Brasseur (1869-1870, 2: 103-110), han sido objeto frecuente de estudio y de traducción por parte de los mayistas. ${ }^{2}$

Cinco son las que cabe denominar fuentes para esta serie de textos mayas oraculares: el Chilam Balam de Maní (CP, ms., 65-75), el de Oxkutzcab (CP, ms.: 166-170), el Chilam Balam de Chumayel (ms.: 104-107), el de Tizimin (ms.: 7r-10v), y Lizana (1633: 61r-64r; 1892: 37r-39r). Salvo los datos introductorios exclusivos del Chumayel (ms.: 103-104; Roys, 1967: 60, 164), la única fuente completa e ininterrumpida para esta serie de augurios se encuentra en el Chilam Balam de Maní (CP, ms.: 65-75). De no haberse extraviado su folio 9, el Chilam Balam de Tizimín constituiría otra fuente completa.

En conjunto, la sección íntegra que contiene las "profecías" comprende casi 462 líneas. Los datos preliminares del Chumayel, donde se da una lista de los augures, abarcan unas 37 líneas; 34, asimismo y de igual contenido, son las del Códice de Maní y del Tizimín. Excluido lo cual, siguen unas 150 líneas de material profético-histórico y, ${ }^{3}$ a continuación, comprendidos en 195 líneas, están los dichos oraculares en sí. La sección se cierra con 42 líneas informativas, donde se proporciona, otra vez, la lista de los profetas ( $C P$, ms.: 74-75).

La naturaleza de estos oráculos no puede, en rigor, llamarse profética. La sensible amalgama de elementos cristianos que acusan las "profecías", atestigua que los presuntos profetas habían estado expuestos a la predicación evangélica. Las "profecías" de Ah Na Hau Pech y de Patzin Yaxum Chan son, más bien, piezas parenéticas. Pero, sin necesidad de invocar la hipótesis de que alguno de estos augurios sea un embuste burdo confeccionado en complicidad con los frailes, cabe atri-

${ }^{2}$ Por mencionar algunos, Barrera Vásquez (1969), Edmonson (1982), Makemson (1951), Mediz Bolio (1930), Solís Alcalá (1949) y Tozzer $(1917,1921)$.

${ }^{3} \mathrm{El}$ que desee formarse una idea general sobre la naturaleza de esta sección, hará bien en leer "The Prophecy of Chilam Balam and the Story of Antonio Martínez", en Roys (1967: 120-125). Es, con algunas variantes, lo mismo que trae el Códice Pérez (1949: 132-146). 
buir la composición de los textos a legítimos ah kinob mayas, que, en la tradición secular de su oficio, continuaron vaticinando la carga de los katunes.

$\mathrm{Al}$ aludir al arribo o al retorno de hombres blancos, $\mathrm{y}$ a ciertas ideas cristianas, no estaban anticipando por inspiración sobrenatural conceptos ni acontecimientos, sino, más bien, dando a los conocidos el cariz que el katún reinante permitía atribuirles. Cuando los pronósticos del katún 13 Ahau fueron formulados, probablemente en noviembre de 1510 , los sacerdotes mayas sabían ya que ese katún concluiría el mes Xul del año 11 Ix. En ese mes, se decía, bajaba Kukulcán, o Quetzalcóatl, a la tierra. Kukulcán, cuyo anunciado retorno por el oriente era esperado y temido. Por lo que un hombre de razón llamaría sólo coincidencia, durante el katún 13 Ahau, final de ciclo, llegaron por el oriente hombres barbados y blancos. Su llegada y el retorno de Kukulcán debieron entrelazarse entonces. Por lo demás, los oficiantes del sacerdocio nativo tenían una marcada tendencia a absorber, de modo sincrético, nociones y ritos de cualquier religión extraña. Los mayas, como los mexicanos, se inclinaron por eso... desde el primer contacto, a una simbiosis cristiano-pagana. ${ }^{4}$

No hay repugnancia, por ende, en que las "profecías" se hayan compuesto, post factum, en pleno tiempo de la conquista española. Ah Kauil Chhel, habiéndose refugiado en Chetumal, declara haber escrito (¿pintado en glifos?) en “...febrero, 15 días de 1544", junto con $\mathrm{Ah} \mathrm{Na}$ Puc Tun, el material del Cuceb (CP, ms.: 115) ${ }^{5} \mathrm{El}$ asedio y conquista

${ }^{4}$ En 1585, Sahagún (“Arte adiuinatoria...”, ms. de la Biblioteca Nacional de México, f. 101v), haciendo un balance finisecular de la predicación evangélica entre los indios de Nueva España, reconocía con amargura el fracaso de ésta:

A todos nos fue dicho (como ya se hauía dicho a los $\mathrm{P}$ [adr]es dominicos) que esta gente hauía venido a la fee de veras, y estauan casi todos baptizados y tan enteros en la fee cathólica de la Iglesia Romana, q[ue] no haúa necessidad ning[un]a de predicar contra la idolatría porq[ue] la tenían dejada ellos muy de veras. Tubimos esta información por muy verdadera y milagrosa porque, en tan poco tiempo y con tan poca lengua y predicación, y sin milagro alguno, tanta muchedumbre de gente se haúa conuertido... Hallóse, después de pocos años, muy euidentem[en]te la falta que de la prudencia serpentina vbo en la fundación de esta nueua Iglesia, porq[ue] se ignoraua la conspiración que hauían hecho entre sí los principales y sátrapas de recebir a Jesuchr[ist]o entre sus dioses como vno de ellos..., conforme a la costumbre antigua que tenían, que, quando venía alguna gente forastera a poblar cerca de los que estauan ya poblados, quando les parecía, tomaban por dios al dios de los rezién llegados... De esta manera, se inclinaron con facilidad a tomar por dios al dios de los hespañoles; pero no para que dejasen los suyos antiguos...

${ }^{5}$ Querría subrayar, sin embargo, que la cronología biográfica de estos profetas es muy incierta en las fuentes, y a veces contradictoria. Más adelante voy a citar el Tizimín 
final de Uaymil-Chetumal se efectuó entre 1543 y 1545 (Chamberlain, 1948: 232-236). Si no fueron capturados allí, es posible que ambos sacerdotes hayan huido, como tantísimos otros, a la isla de Tayasal. Nadie sabe, ni ha quedado constancia de si se llevaron consigo el rollo de sus augurios. En cualquier caso, un fulano o fulanos que habían aprendido a escribir con caracteres fonéticos europeos, reescribieron o reinventaron los textos que han transmitido las cinco fuentes ya mencionadas. David Bolles (ms., s.f.) observa

... that much of the prognosticatory material in the Books of Chilam Balam shows a certain uniformity in writing style (grammar, vocabulary, phraseology, etc.). While it is tempting to ascribe this uniformity to the work of a single person, this uniformity could also be the result of an educational system in which much of the learning was done by rote.

Miguel León-Portilla (1992: 26) opina entretanto que:

La historiografía maya de los chilames... se reescribió sin cesar de esta manera. El registro de aconteceres pasados, de experiencias presentes y de profecías se hizo en papeles que se iban deteriorando con el uso continuo a través de los años. Quienes cuidaban de ellos, le hacían añadidos. Atendiendo a su presente, al de la comunidad entera, modificaban los relatos acerca del pasado y expresaban nuevas profecías. Según éstas se cumplían o parecían posponerse, volvía a modificarse la interpretación de los hechos. Cuando los viejos papeles se desgarraban ya, por haber sido leídos y reescritos en innumerables ocasiones, la antigua palabra se copiaba en hojas nuevas. Así se procedió a través de los siglos, hasta fechas muy recientes.

\section{El ejercicio de la profecía}

El de pronosticar era oficio ancestral ejercido por casi todos los sacerdotes nativos de Mesoamérica. Su ejercicio era, al parecer, responsable, y requería vastos conocimientos de astronomía y teológicos. El tiempo, su transcurrir y sus efectos benévolos o contrarios a los quehaceres humanos se asociaban al carácter moral de los dioses que presidían cada periodo. Cada periodo, hasta los breves del entredía, tenía atributos divinos o cosmológicos. Un complejo tejido de nervios físicos y metafí-

(ms.: 13), donde se afirma que Ah Kauil Chhel, Ah Na Puc Tun y Ah Xupan Nauat fueron sacerdotes en Uxmal, al servicio del halach vinic Hun Uitzil Chac Tutul Xiu, lo cual remitiría sus respectivos florecimientos a unos 100 años antes de la conquista española. Tratándose de la vida de estos profetas, toda cronología debe mirarse con gran reserva. 
sicos era el responsable de los dolores, expectativas y retribuciones terrenales de los hombres. Conocer esta trama, y sus presuntos efectos sobre la naturaleza, constituía, en sus circunstancias y género, una auténtica ciencia. Una ciencia que, por desgracia, la obstinación demencial de unos religiosos aniquiló en muchas partes y, en otras, destruyó la violencia de la conquista castrense.

Los publicados aquí son pronósticos mayas, aunque los ah kinob que los compusieron o declararon hayan estado expuestos, en grado mayor o menor, a nociones religiosas cristianas que ellos no rechazaban. Por el contrario, su acusada tendencia al sincretismo teológico los inducía a aceptar, a transubstanciar, los dioses extraños; su panteón era un centro de convivencia pacífica, no un reino hegemónico, imperial o monárquico. Esa actitud no fue, por supuesto, compartida por los evangelizadores hispanoromanos ni podía ser admitida por la "única religión verdadera".

Paradójicamente, un celoso portaestandarte del catolicismo romano, fray Bernardo de Lizana, contribuyó a difundir esos oráculos mayas bajo la falsa idea, primero, de que eran cronológicamente anteriores a la llegada del europeo a tierras americanas y, segundo y por consecuencia, de que eran "profecías" inspiradas por Dios a los sacerdotes idólatras. Tan descabellada suposición, que aceptó sin protesta López de Cogolludo más tarde, obligó a Lizana (1633: 60r-v) a racionalizar este don concedido por el Único Dios Verdadero a los ah kinob mayas. Su razonamiento teológico, si es que merece epíteto tal, será, para siempre, ejemplo señero del grado de aberración al que cualquier hombre, en posesión de todas sus facultades, puede llegar.

Los cinco oráculos mayas que se ofrecen a continuación fueron fijados, al parecer, entre 1544 y 1548 , cronología que no necesariamente afecta la biográfica de sus presentes autores. La composición de la serie final en sí fue, entretanto, compuesta hacia 1527 , fecha en que el adelantado Montejo efectuó la primera entrada por el oriente de la península yucateca. Dos o tres de dichos augurios, sin más particularidades, dan la impresión de haber sido compuestos en el periodo durante el cual los españoles se retiraron a Tabasco (1529-1535) a preparar la que sería etapa final de la conquista de Yucatán. De ser ése el caso, cabría pensar, entonces, que los augurios no constituyen anticipos proféticos de la "llegada" del hombre blanco con sus cerbatanas de fuego y sus cruces, sino presagios realistas de su inminente regreso a las tierras de Yucatán.

Los mayas sin duda, para esa época, tenían conocimiento de la conquista de México, del istmo de Tehuantepec y de Centroamérica. Un 
anillo de fuego los cercaba por todas partes; la crueldad despiadada de la horda conquistadora era manifiesta: muchos buscarían refugio en Bolonte Uitz, ${ }^{6}$ cerca de los itzaes; pronto se mecería inerte la cola del quetzal, del ratón, quebrada para siempre su rabadilla. Las ominosas señales del katún 13 Ahau se habrían cumplido.

\section{Los augures y sus nombres}

Las fuentes han conservado los nombres de los augures a quien se atribuye haber tenido anticipos de la conquista y evangelización españolas. Tales registros, empero, no son uniformes. Algunos ignoran nombres enumerados en otros, y viceversa, o, sin razón alguna aparente, un par de nombres resultan, al fin de cuentas, inciertos. El Chumayel (Roys, 1967: 164-165, n. 8) y Lizana (1633: 61r) hacen mención de un Ah Kuil Chel que los códices de Maní y Oxkutzcab $(C P$, ms.: 72, 166) identifican como Ah Kauil Chel, y al cual López de Cogolludo (1957: 98) llama Ah Kukil Chel. El nombre que ha sancionado la erudición moderna es el de Ah Kauil Chhel, con fundación en el hecho de que Kauil aparece en una advocación de Itzam Na y es, además, un apellido bastante común en Yucatán (Roys, 1967: 165, n. 5).

Es conflictivo también, más que el anterior, el nombre del sacerdote al que Lizana (62r), el Chumayel (Roys: $164-165,167,186)$ y el $O x$ kutzcab (CP, ms.: 168) identifican como Natzin Yabun Chan; el Maní (CP, ms: 72), como Ah Natzin Yuban Chan; la Crónica de Chhic Xulub Chhen (información de Bolles, 29 de noviembre de 1992), como Nadzimabun Chan; y López de Cogolludo (1957: 97), como Patzin Yaxun Chan. Lo más probable es, al parecer, que este augur se llamara Patzin Yaxum Chan, "el noble Quetzal-Serpiente". El kuk (Pharomacros mocinno) o quetzal aparece comúnmente aparejado con el yaxum (Cotinga amabilis), que era un símbolo de Kukulcán o Quetzalcóatl (Roys, 1967: 74 , n. 7; 99, n. 8); chan es, en este caso, una voz choloide que significa 'serpiente'. Tanto el prefijo Patzin, como el presumible patronímico Chan, son elocuentes indicios de que este augur, si es que existió en efecto, procedía de la religión chontal (véase Scholes y Roys, 1968: 65).

Sobre el nombre de los augures restantes, hay concordia en las fuentes. Tratándose de Ah Na Hau Pech, — “el hijo de madre Hau y de pa-

${ }^{6}$ Inexplicablemente, Ermilo Solís Alcalá (CP, 1949: 133) traduce el topónimo Bolonte Uitz por "nueve puntos en círculo". Véase Chumayel (Roys, 1967: 64, n. 3; 121 , n. $6 ; 139$, n. 5 ). 
dre Pech", nombre que, dada de la escasa confiabilidad de los amanuenses, resulta inquietamente cercano al de Ah Cun Pech, Ah Dzun Pech y Ah Naum Pech (Relaciones histórico-geográficas... Yucatán, 1983, 1: 377; Roys, 1967: 86, 119, n. 2; Tozzer, 1941: 43, n. 216; 98, n. 440)—, dice Roys (1967: 186):

[He] is believed to have lived about four katuns, or eighty years, before the coming of the whites, which would be about the time of the fall of Mayapan. He was probably a member of the powerful Pech family which governmed the Providence of Ceh Pech at the time of the Conquest.

Ah Kauil Chhel debió pertenecer al linaje Chhel, gobernante de la costa nororiental y central desde Dzidzantun y Tecoh. Ah Na Puc Tun, "el hijo de la madre Puc y del padre Tun", pudo ser entretanto originario y sacerdote de Chetumal; pero esta afirmación es incierta. En 1582, un don Andrés Tun gobernaba el pueblo de Kantunil, a escasa distancia de Itzamnal. ${ }^{7}$ De ambos, y de Ah Xupan Nauat (tal vez Ah Xiu Pan Nauat), cuyos antecedentes y filiación pone de manifiesto su nombre, dice el Tizimín (ms.: 13) que fueron sacerdotes del halach vinic Hun Uitzil Chac Tutul Xiu en Uxmal (véase Tozzer, 1941: 36, n. 177). Dato que, de aceptarse, induciría a creer que estos sacerdotes vivieron casi 100 años antes de la conquista.

Por fin, sobre el profeta Chilam Balam, probablemente oficiante en Maní, Juan de Palomar (Relaciones histórico-geográficas... Yucatán, 1: 69) dejó referido que

... Pocos años antes de que los españoles viniesen a conquistar esta tierra, un indio principal, que era sacerdote, llamado Chilam Balam, que le tenían por gran profeta y adivino... les dijo que dentro de breve tiempo vendría, de hacia donde sale el sol, una gente blanca y barbada, y que traería levantada una señal como esta, $\dagger \ldots$ Y mandó al señor de Maní, que se llamaba Moo Chan Xiu, que ofreciese a los ídolos aquella manta para que... quedase por memoria, y aquella señal de la cruz, y otras, hizo hacer de piedra labrada...

${ }^{7}$ Según carta que dirigieron 13 caciques a Felipe II, conservada en el Archivo General de Indias (Audiencia de México, legajo 104), el texto maya está fechado en enero 6 de 1580, sin firmas; la traducción española, hecha por Jhoan Ruiz de la Bega, está fechada dos días después y ostenta las firmas de los caciques. El dato que invoco no garantiza, sin embargo, que los Tun hayan sido señores tradicionales en Kantunil. En la sección de Justicia (245, Contaduría 920) del mismo Archivo, hay otra carta de caciques de Yucatán, por la que consta que en 1565 era cacique de Kantunil Pedro Dzul. 
Roys (1967: 187) opina que,

in Katun 2 Ahau [wich covered approximately the first two decades of the Sixteenth Century] he predicted that in the Katun 13 Ahau following, bearded men would come from east and introduce a new religion.

Hay que tener en cuenta, sin embargo, que Ah Moo Chan Xiu presidió la junta celebrada en Maní en 1537, donde se discutió traer a los extranjeros a dicha provincia, "because the head-chief of the town, Ah $<$ Dzun> Xiu, was killed at Otzmal" (Roys, 1967: 145-146). Parece obvio, entonces, que Ah Moo Chan Xiu asumió el gobierno de la provincia en 1537 como sucesor del asesinado cacique, ${ }^{8}$ y que, dada la finalidad de esa junta y el lugar en que se efectuó, participó en ella Chilam Balam.

Comoquiera que haya sido, el propio augur declara en su oráculo (E/43-45): Licil in binel... $u$ than hahal Ku... tu bolon piz... haabil Ah Hun Ahau katun... [Ahora propago... la palabra del verdadero Dios... en el noveno año del katún de Ah Hun Ahau... ]. No puede tratarse del katún 1 Ahau, porque el primero cayó en 1382-1401; en 1637-1657, el segundo. Debe tratarse, entonces, del katún presidido por el dios infernal Hun Ahau, nombre que recibía el planeta Venus (Thompson, 1960: 218-219), asociado con Kukulcán y el katún 11 Ahau. La profecía en cuanto tal corresponde al katún 13 Ahau; pero pudo quedar fijada en 1548 (=7 Cauac), año en que fue sofocada la última sedición de los mayas. No resulta creíble que un sacerdote maya, $\sin$ haber estado expuesto a las nociones cristianas, hablara de propagar la $u$ than hahal $K u$. Aparte de favorecer la no resistencia ante el extranjero, como es bien sabido, el Chilam Balam de Maní había absorbido, al parecer, una buena dosis de ideas cristianas.

\section{Nuestra versión de las profecías}

La que ofrecemos aquí es una transcripción miscelánea de las profecías mayas, no una edición crítica. Los cambios hechos al texto no se justifican ni se razonan a cada paso en notas al pie de página. La transcripción es producto de un paciente cotejo efectuado en la fuentes. Lizana (1633: 61r-64r) en primer lugar, cuya obra, no obstante haber salido de

${ }^{8}$ Sobre la matanza de Otzmal (1537), perpetrada alevosamente por los Cocom en agravio de los Tutul Xiu, véase Tozzer (1941: 54-55, n. 269). 
la estampa con errores de mucho tomo, registra la copia de las profecías más antigua que se conoce. Después, el Chilam Balam de Oxkutzcab ( $C P$, ms.: 166-170), cuyo manuscrito antiguo, hoy conservado en copia tardía, consultó sin duda Lizana. Le sigue el Chilam Balam de Chumayel (ms.: 104-107) primero, porque sabemos que el manuscrito ahora extraviado databa de 1782 (Roys, 1967: 7); porque es la obra del género mejor conocida entre los estudiosos, y porque ofrece material (de dudosa autenticidad) no contenido en las otras fuentes, y además variantes curiosas. Por fin, de manera un poco arbitraria, concedimos el cuarto y quinto lugar al Chilam Balam de Tizimin (ms.: 10r-v) y al Chilam Balam de Maní (CP, ms: 65-67), respectivamente. En adelante, estas fuentes serán identificadas por las letras capitulares C (Chumayel), L (Lizana), M (Maní), O (Oxkutzcab) y T (Tizimín).

Para efectuar el cotejo, hicimos primero una transcripción diplomática de los textos, la cual ordenamos en cinco columnas paralelas, cada una correspondiente a la obra copiada. El texto, entretanto, lo repartimos en líneas de desigual longitud, procurando, ante todo, destacar la construcción parafrástica de las oraciones mayas y, después, que cada línea contuviera una oración completa, o una subordinada que tuviera sentido. Por efecto de ese artificio, adquirieron los textos una apariencia de estrofas versiculares. En modo alguno ha sido nuestra intención insinuar que las profecías originales se formularon o recitaron así.

Una vez que estuvo construido ese texto matriz a cinco columnas, procedimos a componer la versión ofrecida aquí. El procedimiento fue doble. Las variantes que no funcionaban en la frase gramatical fueron eliminadas y, por natural consecuencia, fueron seleccionadas aquellas que sí conformaban un discurso maya gramatical, sin violentar el universal del contexto. Un par de ejemplos ilustrarán el método empleado mejor que largas explicaciones. Sean las líneas 3 y 2 de las profecías de Ah Kauil Chhel y de Patzin Yaxum Chan, respectivamente, cuyas variantes seleccionadas marcaré con negritas en el cotejo.

L valac utal Macbin cadzab tocodz pop Katune

O ualac u tal Mac bin ca dzabto codz pop katune

C ualac u talel: mac bin ca dzab tu codz pop: katune; yume

T [folio 9 extraviado; laguna]

M Ualac u tal el, mac bin ca dzabac tu coodz poop katune

B/3 Ualac u talel, mac bin ca dzabac tu codz pop katune, yume?

L Yah kinobuil bin puch cob vchmal

O Yah kinob uil bin puch cob uchmal 
C Yah kinob uil bin puch cob uchmale=

T Yah kinob va bin pudz cob vch mal

M u yah kinobi uil bin pudzcob tu kinile

$\mathrm{C} / 2 \mathrm{U}$ yah kinob iuil bin pudzco< $<>\mathrm{b}$ tu kinile

En su traslado y traducción del Chumayel, Roys (1967: 60-61, 165, 167) lee y traduce, respectivamente:

1. $\quad[\ldots]$ mex ca a nate ualac

2. u talel. Mac bin cadzab tu codz pop katune, yume?

[...] ye shall not understand when it comes.

Who shall believe it at the rolling up of the mat of the katun?

2. [...] yah kinob uil bin puchcob uchmale.

[...] of this priests who will bring it in time to come.

Y, curiosamente, explica en la nota 1 (p. 167): "Here the Maya puch$c o b$ has been translated as though it were pulicob". Pero no explica de cuál de sus mangas se sacó el pulicob, ni sobre cuáles bases está fundada la elección de ese verbo.

En algún caso sin embargo, por fortuna aislado, nuestra "reconstrucción" tuvo que ser atrevida. Me refiero a la línea 2 del profeta $\mathrm{Ah} \mathrm{Na}$ Puc Tun, que es, salvo juicio menor, una de las difíciles. La secuencia textual es como sigue:

\section{Ox vahom Kauil va ah tan vchmal \\ O Oxuahon kauil, uahtan uchmal \\ C V uaom kauil; ua ahtan u chamal \\ T [laguna] \\ M oxuahom kauil uuan ahtan uchmal}

A/2 Ox<0 $>$ uahom Kauil $\mathrm{u}$ ua $<\mathrm{a}>\mathrm{n}$ ah $\tan$ uchmal

Roys $(1967: 60,165)$ traslada y traduce:

2.

U uaom Kauil;

3. ua ah $\tan$ uch[a]amal.

Kauil shall be set up; he shall be set up in front in time to come.

La construcción maya parafrástica del contexto fue bien aprehendida por él, pero en este caso no funciona gramaticalmente; su traducción es 
de tanto sabor antiguo, que hasta sugiere la erección de una estela en honor de Kauil, quienquiera que el personaje haya sido. Traducir ua ah tan por "he shall be set up in front" es, empero, una licencia inadmisible. La profecía, por lo demás, habla de fuego sobre la tierra, de miseria y trabajos (numya) que se avecinan. Una especie de glosa, contenida en el prefacio de las profecías (C 104), dice:

Sac petahom Canal $=$ elom ti $\mathrm{cab}=\mathrm{ychil} O \mathrm{x}$ ahau Katun hun Ahau Katun $=\mathrm{V}$ lobil ox dzit Katun $=$

[Se formará una anillo blanco en el cielo; arderá la tierra. En el katún 3 Ahau $<y>$ el katún 1 Ahau, está contenida la maldad del tercer katún]. ${ }^{9}$

Con lo anterior no se pretende, ni por asomo, insinuar que nuestra "reconstrucción" y traducción de los textos sean inobjetables y perfectas. En tanto que la primera selecciona variantes, depende de apresuramientos y falibilidad de criterio; en la medida en que la segunda interpola elementos gramaticales o sintácticos, está limitada por el conocimiento individual que tenemos de la lengua que hablan ahora, o hablaron antiguamente, los mayas de Yucatán. Tampoco hay que descontar los presumibles errores de transcripción de las fuentes y los errores involuntarios de mano del transcriptor.

Hay líneas entretanto cuya reconstrucción continuará incierta hasta que se descubran nuevos elementos de juicio. Los manuscritos mayas sobrevivientes son, tratándose de este caso, guías falaces. Son palabras nuevas escritas sobre palabras antiguas. Los prejuicios y vicios de pluma del último escriba borran y desfiguran las palabras originales. La autoridad con que pesan las opiniones de algunos reputados especialistas, también contribuye a paralizar la creatividad potencial del nuevo investigador. Hay líneas que pocos se atreverían a retocar, por aquello de que ya el magister dixit. ¿Cuántos se atreverían, para traer un caso, siquiera a poner en duda este encabezado: "The prophecy of Chilam Balam, the singer, of Cabal-chhen, Manr'” (Roys, 1967: 167)? Véase sin

${ }^{9} \mathrm{La}$ última frase parece hermética; pero, si se acepta su consecuencia, debe estar refiriéndose al katún 12 Ahau, cuya ocurrencia, después de la conquista, tuvo lugar en 1677. Podría, entonces, ser anticipo de lo que el maya habría de padecer a finales del siglo XVII, o una explicación de lo que estaba sufriendo ya en las últimas décadas de ese siglo. Obsérvese el uso de la partícula dzit para contar los katunes. El CMM (1984: 131r) la define como "cuenta para candelas, hebras de hilo o seda, varas delgad[a]s". Roys (1967: 164-165) traduce las líneas mayas citadas: "There shall be a white circle in the sky. It shall burn on earth in Katun 3 Ahau, in Katun 1 Ahau, the worst of three katuns". 
embargo el breve comentario E/0, que en ese lugar ilustra los problemas que entraña adoptar dicho encabezado.

No está de más aclarar, por fin, que los textos oraculares mayas que están a continuación se identifican con letras capitulares. Van de la $\mathbf{A}$ a la E. Es para combinarlas con los números marginales de la línea (de 0 en adelante), y evitar así las llamadas dentro del texto. $\mathbf{A} / 0$, por ejemplo, sería la nota al pie correspondiente al encabezado de la profecía de Ah Na Puc Tun; C/8, la nota que corresponde a la línea 8 de la profecía de Patzin Yaxum Chan, etc. La ortografía maya que empleamos, es la siguiente:

$$
c h h=c^{\prime} ; d z=0 ; p p=p^{\prime}
$$

Para cerrar estas páginas, me agrada reproducir unas declaraciones de Bolles (a Acuña, 3 de abril de 1992), que gustoso suscribiría:

When I did the translation of the prophecy of Chilam Balam some time ago, I was struck by the fact that I had a really very difficult time in translating it at all. Once again, while working on the translations of the other prophecies, I must say that I am really still not adequately prepared to make these translations in a way that I would like to be able to do. The truth is that there is so much more which has to be done in terms of getting all the available information from the various dictionaries in place, so that the meaning of every word and, just as important, the meaning of every phrase is readlily apparent... What I have done up until now is to go through all of the texts and made translations in more or less a preliminary manner... and trying to make sure that my English really says what the Mayan text is saying. I must say, though, that some times that is close to an impossible task.

Al leer la última frase, muchos habrán recordado el malicioso humor con que Thompson, en su semblanza de Roys (1967: vii), comentó el asunto:

When it came to writing Maya manuscripts, the Maya scribes of the colonial period played poker with deuces wild. Their punctuation was their own and their division of words would have made Noah Webster take to the bottle as readily as did the original Noah when he went ashore on Mount Ararat... 


\section{LAS PROFECIAS MAYAS}

\section{A/U than Ah Na Puc Tun, ah kin}

Elom ti cab, zac petahom ca<a>nal; $o x<o>$ uahom Kauil $u$ ua $<a>n$ ah tan uchmal. Elom ticab, elom dzidz tu Katunil uchmal. Talome u pixan.

5 Bin ilic, bin yalic $u$ than, bin yokte u numyail; u ppitic numyae u pixan, bin kamic u macul, u ppitic numya lae.

[Palabra de Ah Na Puc Tun, sacerdote]

Habrá fuego en la tierra, un círculo blanco habrá en lo alto; abrasará Kauil con su vaho lo que se yerga a su paso en el porvenir.

Ha de arder la tierra, se abrasarán los cascos en el katún que va a entrar. Palparse ha, sin falta, su suerte.

5 Verse ha, se pronunciará su palabra, se llorará su miseria; levantará miseria su suerte, recibirse ha su paga, se elevará la miseria.

\section{B/ U than Ah Kauil Chhel, ah kin}

$\mathrm{Cu}$ hidzib te katun, yume, maix ca a naate.

Ualac u talel, mac bin ca dzabac tu codz pop katune, yume?

Bin uluc tu cal ya.

Tali ti xaman, tali ti chikin.

5 Tu kinil iuil yanom, mac to ah kin, mac to ah bobat bin alic $u$ than uoohe ichil Bolon Ahau uale?

Maix ca a naate hunac tzuc ti cab uale.

Hun dzal pi<c tan yo $>$ nel tzutzuc Chac.

Bay ci oltzil iuil ah tepal cuchie,

10 cii uil yokol yahaulil cabobe.

Kah cun au ol, ah itzae.

[Palabra de Ah Kauil Chhel, sacerdote]

Cuando concluya el katún, oh padre, no te darás cuenta tú.

Cuando venga, ¿quién pondrá entonces en la estera arrollada al katún, oh padre?

$\mathrm{Va}$ a venir con recio dolor;

$5 \quad$ va a llegar por el norte y por el poniente.

Cuando ese momento sea, ¿qué sacerdote, qué profeta declarará el mensaje de la letra contenida en Bolon Ahau?

Tal vez nada entiendas en todo el mundo. 
Una simple presión de mano equivale a muchos parientes del deshonesto Chac.

10 Como el oropel del que fue gran señor, así el placer del que está sobre los reyes del mundo.

¡Grábalo en tu memoria, ah itza!

C/ $U$ than Patzin Yaxum Chan, ah kin

Uchi $u$ than hahal Ku ti peten, lay $u$ pakte $u$ hokole, yum. U yah kinob iuil bin pudzco $<o>b$ tu kinile,

5 A pixaneex bin hahal kamice. dzaex ka a naateex tu than, tu tzacil kae.

Xeth a uol ta kul, ah itzae;

Tubez a hauay kue, a zatay kue, lay a kul te u hahal Ku lae tu lacal yanil ah Tepale, yume.

10 Yah chhab uil ti bal cah tu zinil. lay ya ta uol in ualic teche, maya ah itza. Ma a kat a uuy u yanal dios.

"Hah a kul", ta thano, lay u yocol tun ta uol tu than in tzeec lae.

[Palabra de Patzin Yaxum Chan, sacerdote]

Antiguamente existió la palabra del verdadero Dios en la provincia, el cual por todos era esperado, oh padre.

Sus sacerdotes tal vez fueron ahuyentados entonces, dejándoos entendidos en su palabra, en su historia.

5 Vuestras almas, dicen, de verdad lo aceptaron.

Aparta el corazón de tu ídolo, oh ah itzá;

olvida tus ídolos perecederos, mortales;

esto es, adora al verdadero Dios, que

es el supremo Señor de todas las cosas, oh padre.

El Creador ha venido al mundo por todas partes,

lo cual te causa pesar cuando te lo digo, oh maya ah itzá.

Rehúsa dar oídos en adelante a otro dios.

"Verdadero es tu dios", dices tú;

esto es, que entra ya en tu corazón la palabra del que predicó.

D/ U than Ah Na Hau Pech, ah kin

Tu kinil uil u naatabal ah kine, yume, ti yokzah ich ah tepal uale.

Can dzit $u$ katunil uchomi uale u hahal pul tu kin Kue.

$5 Y$ Yok tah ba, kay ba, in kubene, yume. Ti a uicheex tu beel a uulaex ah itzae.

U yumil cab ca ulom; 
ca dza a uol:

ti tali tu chi Ah Na Hau Pech, ah kin.

Tu kinil uil Can Ahau katun uale, tu hidzil u katunil, yume.

10

Zatom uiil. Zinic uinicil bin yanac tu pach uiil, tu men u bobochil uiil, u chhuyum thulil uiil, zinic, dziu, kau, pichh, ix pucil.

[Palabra de Ah Na Hau Pech, sacerdote]

A su tiempo será entendido el augur, oh padre, cuando se apiade el Supremo Señor.

Tal vez cuatro cuentas del katún habrán de pasar para que venga la verdadera noticia de Dios.

5 Ruega, alza la voz: es mi encargo, oh padre.

A vuestros ojos está el camino de vuestros huéspedes, oh ah itzá.

El señor del mundo habrá de volver sin falta; préstale atención:

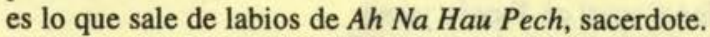

En el tiempo del katún 4 Ahau,

tal vez sea el fin de los katunes, oh padre.

10 Habrá de desaparecer la comida. Huevos de hormiga serán el alimento postrero,

a causa de la voracidad del boboch, del gavilán,

de la hormiga, de los sanates, grajos, pajaros mosca, ratones.

E/ U than Chilam Balam, ah kin de $<T>$ ixcoyom

Ca uichen mani Oxlahun Ahau, $u$ hidzil uil katun;

ualac uil Itza, ualac uil Tancahe, yume,

u chicul Hunab Ku caanal.

Ulom uaom che; etzahom ti cahe

5 uchebal u zazhal yokol cabe, yume.

Dzuni moc tan ba, dzuni zauinal;

ca talom ti pul chicul Ku uchmal ah kin, vinice, yume.

Hun auat, hun lub u tal.

A uiliceex mute u tippil yetel uaom che.

10 Ahom cab hun xaman, hun chikin;

ahom Itzam Na Kauil.

Talel $u$ cah ca yum, ah Itza; talel u cah ca zucun, Tantune.

Kam a uulaob, ah mexoob, ah likin caboob,

ah puloob tu chicul Kue, yume.

$15 U$ Utz tun ua $u$ than $K u$ cu talel cicnale?

Talel $u$ cah $u$ kin ca cuxtale.

Ma a zatic uay yokol cabe, Yume,

tech Hunab Ku, chhab ti coon.

Utz tun ua u than $K u$, yume, yah canul ca pixan?

20 He mac bin kamic hach ocaan ti yole

ti caan $u$ bin tu pach;

heuac u chun ca kin uinicil.

Ca ua cun to u chicul caanal;

ca ua cun to ca pacte hele; 
ca ua cun to u uaom che.

Num tetah u kexac hokol helela:

$u$ hel tu pach $u$ Yax Cheil Cab etzahom helel ti bal cahe.

Lay u chicul Hunab Ku caanal, lay a kul teex, ah Itzaexe.

Ca a kul te hele u chicul kulil Ku caanale;

$0 \quad$ ca a kul te to tu hahlil oc olal;

ca a kul te to ca hahal Kue.

Ocez ta uol tu than Hunab Ku.

Tali ti caan a uah thanule.

Cux cin ta uol, ah Itzaexe: ahom uil cab tiob.

35 Oczicoob ti yol ichil u yanal katun uale.

Yaab tu ba in than. Cen Chilam Balam.

Ca tin tzolah $u$ than hahal $\mathrm{Ku}$ tu zinile yokol cabe.

Licil in binel hunac tzuc ti cab

$u$ than hahal $K u$, yumil caan yetel luum,

tu bolon piz haabil Ah Hun Ahau Katun uale.

Hach utz ua u than ti caan, yume?

Cokol yahaulil, cokol ix ca pixan hahal $\mathrm{Ku}$;

heuac ti ukez lae, yume.

Ox alam ukil, ix cuch luum idzinil.

45 Dzaman yol! Cimen ix u puczikal tu nicteob xan

ah uaua tulupoob, ah uatan zinaob

Nacxit Xuchit tu nicte u lakoob.

Ca kin yahauliloob:

coilac te tu dzamoob; coilac te tu nicteob.

Ca kin uinicil cu thanoob;

ca kin u xecoob, u luchoob, u ppocoob.

$U$ coil kin, u coil akab, u maixiloob yokol cab.

Kuy u cal, mudz u uich, pudz u chi

ti yahaulil caboob, yume.

55 Hee cu talel. $M<a>n a<a>n$ hah tu thanoob.

$U$ dzuliloob cah bin yaloob hach talaniloob

$u$ mehen Uuc Tocoy Naob, yaloob Uuc Tocoy Naob, yume.

Mac to ah bobat? Mac to ah kin

bin tohol can tic $u$ than uooh lae?

[Palabra de Chilam Balam, sacerdote de T>ixcoyom]

Cuando veas que pasó el $13 \mathrm{Ahau}$, será el último día del katún.

Manifestará Itzá, manifestará Tancah, oh padre,

el signo de Hunab $K u$ en lo alto.

Habrá de volver la cruz, se hará visible en los pueblos, para que se haga luz sobre el mundo, oh padre.

Ya comenzó la enemistad, ya comenzó la envidia;

vendrán al rechazar el signo de Dios los sacerdotes futuros, la gente, oh padre.

A un grito, a una legua están de venir.

Observad el ave posarse junto a la cruz.

10 Habrá de bajar por el norte, por el poniente;

va a despertar Itzam Na Kauil:

ya está viniendo nuestro señor oh ah itzá;

ya está viniendo nuestro hermano mayor, oh Tantun. 
Recibe a tus huéspedes, los barbados, los de las tierras de oriente,

los que traen a cuestas el signo de Dios, oh padre.

¿Será buena la palabra de Dios que viene a nosotros?

Ya está llegando el día de nuestra vida.

No vayas a desaparecer aquí de sobre la tierra, Señor;

eres Hunab $\mathrm{Ku}$, creador nuestro.

¿Será buena la palabra de Dios, oh padre, que es el guardián de nuestras almas?

20 Cualquiera que reciba la verdadera fe,

al cielo ha de ir con él;

pero el principio de nuestro día está en embrión.

Pongamos en alto, entonces, la señal del cielo;

pongamos en alto, entonces, nuestros ojos ahora;

25 pongamos en alto, entonces, la cruz.

Todo estaba predestinado para cambiar de revelación ahora:

el sucesor del Primer Árbol de la Tierra habrá de mostrarse hoy al mundo.

A este signo de Hunab $K u$ en lo alto, a éste adorad, ah Itzá.

30 da culto a la verdadera fe;

da culto a nuestro verdadero Dios.

Ten fe en la palabra de Hunab $K u$;

venido es del cielo el derecho de tu vida.

Aconsejad bien a los itzaes: podría amanecer para ellos.

35 Haz que entren de corazón en el nuevo katún desde hoy.

Grande por sí es mi palabra. Soy Chilam Balam.

Yo interpreto la palabra del verdadero Dios en toda la extensión de la tierra.

Ahora propago en todo el mundo

la palabra del verdadero Dios, señor del cielo y de la tierra,

40 en el noveno año del katún del señor Hun Ahau en curso.

¿No es en extremo buena la palabra del cielo, oh padre?

Sobre nosotros reina, sobre nosotros y nuestras almas, el verdadero Dios; pero vuélvete a Él, oh padre.

Tres son los hijuelos del piojo, y culpa a la tierra el hermanito menor.

¡Arrepiéntase! Muerto está su corazón de lujuria, y también

el de los que se echan atrás, el de los que sirven, echados,

a Nacxit Xuchit para lujuria de otros.

Son los que dos días gobiernan:

desvergüénzanse allí en sus juntas; desvergüénzanse allí en sus pecados carnales.

50 Hombres de un par de días los llaman; tronos, jícaras, sombreros de un par de días.

Locura del día, locura de la noche son sus bellaquerías sobre la tierra.

Tuercen sus cuellos, guiñan los ojos, fruncen sus bocas

a los que gobiernan la tierra, oh padre.

55 Atranca la puerta cuando vengan. No hay verdad en sus palabras.

Los encomenderos del pueblo van a decir muchas cosas misteriosas

al hijo de Uuc Tocoy Naob, a la hija de Uuc Tocoy Naob, oh padre;

pero, ¿quién va a ser el profeta?, ¿quién el augur

que de verdad explique el significado de estos signos escritos? 


\section{Notas a las Profecías Mayas}

A/1) L Elomticab peta hom canal; O Elom ti cab zac petahom canal; C Elom ticab: petahom canal; M Elon ti cab, zac petahom canal. Esta predicción, observa Roys (1949: 166, n. 22), "is [sometimes] associated with the end of the world in a Katun 13 Ahau" [Roys, 1967: 162].

A/2) La referencia es posible que sea a Itzam Na Kauil. Véase E/11. Sobre el verbo oxou tah, sinónimo de ouox tah, véase la entrada "Dañar con bao o bapor" en el Bocabulario de Mayathan (p. 236) y, en el Diccionario [Maya] de San Francisco (p. 277), oxou. El Calepino Maya de Motul no registra esta voz, pero define ouox tah como 'abahar, echar baho sobre alguna cosa; dañar con el baho o vapor" (352v).

A/3 \} L Elomticab elomdzip...; O Elom ti cab elom dzip...; C Elom ti cab: elom dzidz:; M Elom ti cab, elom ti dzib...

A/4) L ...tu Katunil vchmaltalome/ Vbixanbin...; O ...tu katunil uchmal talome U pixan; $\mathbf{C}$ tu katunil: $\mathrm{u}$ chamal talome $=\mathrm{v}$ bixan...; $\mathbf{M}$ tu katunil uchmal talome, $\mathrm{u}$ pixan...

$\mathbf{A} / 6\}$ Estas dos líneas finales únicamente son registradas por $\mathbf{M}$.

B/2\} L MexKaanaate valac utal; O Mex ka a naate ualac u tal...; $\mathbf{C}$ mex ca anate ualac u talel:; $\mathbf{M}$ maixtan a nate. Ualac u ta lel...

B/6-7\} L Tukinomuil yane/ Macto ahKinbonat/ Bintohalic vthan voohe/ ychil balana Ahau; $\mathrm{O}$ Tu kin nom uil yane Mac to ahkin bobat Bin to $\mathrm{h}=$ alic $\mathrm{u}$ than uoh Ychil balon ahau; $\mathbf{C}$ tu Kinob uil yane yume: mac to ah Kin; mac to ah bouat; bin tohalic uthan uoohe $=y u m e=y$ chil Balam ahau; $\mathbf{T}$ [laguna] uil yane, $\operatorname{mac}$ to ah kin ah bo bat, bin toh alicuthan voohe, ychil bolon ahau uale; $\mathbf{M}$ tu kin iuil ya none: maci ahkin, ahbobat bin alic u than. Uoohe ichil bolon ahau uale... El katún 9 Ahau comenzó en agosto de 1559. El augurio de Ah Kauil Chhel pudo ser pronunciado, por tanto, al mediar el katún precedente, 11 Ahau, en 1549.

B/9\} Estas cuatro líneas finales son registradas sólo por C, y están identificadas en el ms. por los números 7 al 10 (pp. 104-105). Como la esquina derecha inferior de la plana fue severamente dañada por la humedad, la fotografía no registró las letras, muy pocas, que existen entre "hun dzal pi...", final de la línea 7, y "nel", que inicia la línea 8. La transcripción reconstructiva de Bolles es "hun dzal pi $<\mathrm{c}>\tan \langle b i>n$ nel/<be $>$ nel/ $<$ ue $>$ nel/<uo>nel tzutzuc chac". A su juicio, "<it> appears to be a V... the first... of these washed letters" (carta, 4 de noviembre de 1992). Mi reconstrucción y traduccion de "Hun dzal pi<c tan yo>nel..." son sólo discrecionales. Véase tzutzuc, onel y pic tan en el Calepino Maya de Motul (fols. 122r, 351r y 375r). Roys (1967: 60-61) transcribi6: "Hun dzal pitan.../ nel tzutzuc chac", y se limito a traducir tzutzuc chac, "shall be cleansed of shame" (1967: 366 y n. 1).

B/10\} Cii oltzil, que yo he traducido por oropel, suele tener la acepción de "cosa alegre, placentera, agradable y deleitable a la vista"; cit oltzilil es la "beldad y hermosura de cosas vistosas" (Calepino Maya de Motul: 74r).

C/0\} Éste, salvo juicio mejor, no parece un augurio maya auténtico. Es, simple y llanamente, una pieza parenética. Roys (1967: 186), refiriéndose al presunto autor de esta "profecía", declar6: "Nothing is known of Natzin Yabun Chan to the translator".

C/11\} Estas cuatro líneas finales, sólo en $\mathbf{C}$ (números marginales 7 a 10, p. 105).

C/12 C maakat auuy yanil Dios= (línea 8, p. 105). Véase uyah y $u$ yanal en el Calepino Maya de Motul (fols. 447r-v). 
D/9\} Esta fecha no parece aceptable. Katunes 4 Ahau hubo dos inmediatos: uno, a mediados de junio de 1500; el otro, hacia mediados del siglo xvm.

D/10) Estas tres líneas finales sólo en $\mathbf{C}$ (números marginales 6 a 9, p. 105), que lee "Satomuil si/nic= vinicil= bin yanac tu pach uill= tumen $\mathrm{v}$ bobo/chil vijl= $\mathrm{v}$ chu yum thu lil viil $=$ sinic $=$ dziu $=/$ Kau $=$ pichh $=X$ pucil=". Roys $(1967: 166)$ traduce: "The food of the ant<-like> men shall be destroyed..." Véase vinic hal en el Calepino Maya de Motul (fol. 449r) y en Diccionario [Maya] de San Francisco (p. 385).

E/0\} Un fino estudio de esta "profecía", que incluye el cotejo de T, C, y L, en Tozzer (1977: 120-135). En el encabezado de C se lee: "Profeciado de chilam balam; de xi [testado] Zixcoyom-/ [renglon en blanco]/ Cauichen many= Oxlahun ahau vhijdz..." Roys (1967: 61, 167, n. 4), salvo juicio mejor, transcribio y tradujo estas primeras líneas de manera muy objetable. El ms. con claridad indica que este Chilam Balam era "ah kin de $<T>$ ixcoyom" y, tras dejar un renglón blanco, comienza el texto profético con la formula: "Cauichen many=". Que pueden existir otros errores en el deletreo del topónimo Tixcoyom es asunto aparte. En cualquier caso, es de rigor cotejar este encabezado con el que traen las otras fuentes: $\mathbf{L}$ Profecfas de Chilamcalam de Zixcayon cauichen may; O Profecías de Chilam balam; $\mathbf{T}=\mathrm{V}$ profesia chilam Balam, tix kayom cabal chenmani=; M U THAN CHILAM BaLAM AH KIN MANI Pero, si se adopta la lección del T, admito no comprender cómo traduce Roys tix kayom por "the singer". Dato interesante complementario es que el ms. del Chumayel perteneció al maestro de coro de Maní (Barrera Vásquez y Morley, 1949, 48: 14).

Aunque equivocada, ya que empieza en la página 106 por número marginal 4 , cuando debería ser 3, la numeración de las líneas es bastante curiosa en C. Consta de tres series. Una, que va hasta "V chun ca kin uinicil", de 18 líneas; la segunda, que arranca con "Caua cunto $v$ chi cul canal=", corre hasta el número 20 , y la tercera, desde "coylac te tu nicteob=" hasta el final, es de 10 renglones. Esta profecía merece un estudio aparte.

E/1) En efecto, el día 13 Ahau era último día del katún $13 \mathrm{Ahau}$, con el cual se cerraba la rueda de los katunes. El katún 11 Ahau comenzaría en el día 1 Imix. Los indicios apuntan, pues, a que este augurio fue pronunciado hacia 1529.

E/12\} Sobre Tantun Cuzamil, véanse pasajes paralelos en 85 C y 88 C (Roys, 1967: $53-54,145,149)$.

E/17-18\} No resulta claro por qué Roys (1967: 168) traduce estas líneas "You do not fear the world, Lord, you are the only God who created us". Lo que desorienta un poco, sin duda, es la interpelación Yume, aquí al parecer dirigida a Dios.

E/22\} Aquí concluye la primera serie de 18 renglones numerados en el C.

E/26) El verbo num tetah ha producido perplejidad a los traductores. Se compone del prefijo num, que, "en composición", hace extensiva o se intensifica la acción y de tet, de donde el pretérito tetah, que significa 'estar predestinado a' (véase Calepino Maya de Motul: fols. 335v, 417v-418r). Num akab, por ejemplo, significa "toda la noche, o todas las noches"; num caan $U$, "luna llena que dura toda la noche".

E/27] U hel tu pach es, sencillamente, "el sucesor" (véase Diccionario [Maya] de San Francisco: 136). Sobre el yax cheel cab, véase Roys $(102$, n. 2).

E/33\} Esta línea ha sido lef́da e interpretada de muy diversas maneras por los traductores. Sin duda alguna, es difícil. Véase Roys (1967: 168, n. 11) y Tozzer (1977: $127, \mathrm{n} .1$ ). Mi interpretación se basa en que thanul expresa "lo que le es dado a uno, le pertenece o corresponde por derecho" (véase Calepino Maya de Motul: fol. 432r).

E/38-39\} Benel pectzil o Benel than, sinónimos, significan "cundir la fama" (véase Calepino Maya de Motul, fol. 50v; Diccionario [Maya] de San Francisco: 27). 
E/40\} Es posible que, por ser Hun Ahau el nombre de Venus en cuanto dios del inframundo, el cual a su vez estaba asociado con Quetzalcóatl o Kukulcán (Roys, 1949: 159-160; Thompson, 1960: 172), el katún se llamara asf. No me atrevo, sin embargo, a identificar con certidumbre el katún en cuestión. Los 1 Ahau katunes más inmediatos cayeron, uno, en 1401; en 1658, el otro. El noveno año del katún 13 Ahau estaba corriendo entre 1527-1528; el correspondiente del $11 \mathrm{Ahau}$, entre 1547-1548. Esta línea aparece en el Tizimín (7r-10v) y en el Códice de Maní (CP, ms.: 71).

E/41) A partir de aquí, las líneas proceden de C (línea 14 de la segunda serie, pp. 106-107). Salvo juicio mejor, su contenido sugiere que fueron interpoladas por el compilador de esa fuente.

E/44\} El "ox alam ukil= xcuch lum ydzinil..." se lee muy claro en el ms. (líneas 16-17); pero, en el contexto, resulta una insensatez. Yo me inclinarfa a sugerir la lección "ox alam U<l>il...", que fue gobernante en Itzamal. Véase Roys (1967: 178).

E/49\} Con coilac te tu dzamoob concluye, con número marginal 20, la segunda serie de renglones numerados, y comienza la tercera que correrá hasta 10.

E/55\} Véase hee.ah en el Calepino Maya de Motul: fol. 205v. La interpretación de esta frase varía conforme a los traductores. C ...Minan/ hah tu thanob, $v$ dzuliob cah= (líneas 5-6 de la tercera serie, p. 107).

E/56\} Dzulil, dice el Calepino Maya de Motul (fol. 132v), "llaman los indios al encomendero de algún pueblo". Es posible que, antes de quedar cristalizado en esa acepcion, el epíteto se aplicara a cualquier extranjero que viviera a costillas de los nativos de la región.

E/57\}Uuc Tocoy Naob, "las siete casas desiertas".

\section{Bibliografia}

Advertencia: Un asterisco (*) frente a la entrada significa que el documento ha sido consultado sólo en copia parcial; dos asteriscos (**), que su contenido o su descripción son conocidos sólo por referencias.

\section{Documentos de archivo}

**AGI, Justicia 247, México 920

1565 Carta de unos caciques de Yucatán haciendo peticiones al rey.

AGI, Audiencia de México, legajo 104

1580 Carta de 13 caciques de Yucatán recomendando al gobernador on Guillén de las Casas. El texto maya está fechado en enero 6; la traducción española, hecha por Jhoan Ruiz de la Bega, en enero 8. Existe fotocopia en el archivo del Centro de Estudios Mayas.

BoLles, David

1992 Cartas a René Acuña: 3 de abril, 23 de septiembre y 29 de noviembre.

*Códice Pérez

1870 "Chilam Balam. Artículo y fragmentos de manuscritos antiguos en lengua maya colectados y copiados en facsímile por J. P. 
Pérez". Ms. (en $4^{\circ}, 200$ pp.), núm. 50, copiado por Hermann Berendt, en la Berendt Linguistic Collection, Museo de la Universidad de Pennsylvania. Véase Solís Alcalá, Ermilo, 1949.

*Chilam Balam de Chumayel

s.f. El ms. original (en $4^{\circ}$ pequeño, $107 \mathrm{pp}$.) se ha extraviado. Se ha publicado facsímil. Véase Gordon, 1913.

*Chilam balam de Maní

s.f. Véase Códice Pérez.

*Chilam Balam de Oxkutzcab

s.f. Véase Códice Pérez.

*Chilam Balam de Tizimin

s.f. El ms. original (en $4^{\circ}, 52 \mathrm{pp}$.) al parecer se ha extraviado. Existe copia hecha por Berendt en 1868 , ms. núm. 49 , en la Berendt Linguistic Collection. Véase Edmonson (1982), Makemson (1951) y Tozzer $(1921,1977)$.

\section{Publicaciones}

BARRERA VASQUEZ, Alfredo, y Sylvanus G. MorLeY

1949 "The Maya Chronicles", en Contributions to American Anthropology an History, 10/48: 3-85. Pub. 585 de la Carnegie Institution of Washington.

BARRERA VÁsquez, Alfredo, y Silvia RENDÓN

1969 El libro de los libros de Chilam Balam, México, Fondo de Cultura Económica, Biblioteca Americana, serie de Literatura Indígena.

1969 Bocabulario de Maya Than, Codex Vindobonensis N.S. 3833, facsímil y transcripción crítica anotada y edición de René Acuña, México, UNAM, Instituto de Investigaciones Filológicas, Centro de Estudios Mayas. (Fuentes para el Estudio de la Cultura Maya, 10.)

**BRasseur de Bourbourg, Charles Etienne

1869- Manuscrit Troano. Études sur le système graphique et la langue des 1870 Mayas, 2 vols., París, Mission Scientifique au Mexique et dans l'Amerique Centrale.

Ciudad Real, Antonio de

1984 Calepino Maya de Motul, edición de René Acuña, 2 tomos, México, UNAM, Instituto de Investigaciones Filológicas.

Códice Pérez

1949 Véase Solís Alcalá, Ermilo (1949).

\section{Contributions}

1949 Contributions to American Anthropology and History, Washington, D. C., Carnegie Institution. (Pub. 585, núms. 48-51.) 
Chamberlain, Robert S.

1948 The Conquest and Colonization of Yucatan. 1517-1550, Washington, D.C., Carnegie Institution. (Pub. 582).

Diccionario [maya] de San Francisco

1976 Diccionario de San Francisco, edición de Oscar Michelon, Graz, Akademische Druck-u, Verlagsanstalt. (Bibliotheca Linguistica Americana, vol. II.)

**Edmonson, Munro S.

1982 The Ancient Future of the Itza. The Book of Chilam Balam of Tizi$\min$, Austin, University of Texas Press.

*Gordon, George Byron

1913 The Book of Chilam Balam of Chumayel, con una introducción, Filadelfia, Anthropological Publications of the University of Pennsylvania, vol. 5 .

LANDA, Diego de

1941 Landa's Relación de las cosas de Yucatán, a Translation, editado con notas de Alfred M. Tozzer, Cambridge, Massachusetts, Harvard University, Papers of the Peabody Museum, vol. XVIII.

1959 Relación de las cosas de Yucatán, introducción de Ángel María Garibay K., Editorial Porrúa, México. (Biblioteca Porrúa, 13.)

LEÓN PorTILla, Miguel

1992 "Encuentro de Dos Mundos", en Estudios de Cultura Náhuatl, 22 (UNAM): 15-27.

LizanA, Bernardo de

1633 Historia de Yucatán... en Valladolid, por Gerónimo Morillo.

1892 Historia y conquista espiritual de Yucatán, Imprenta del Museo Nacional, México. [Advertencia, no firmada, de Francisco del Paso y Troncoso, autor del traslado diplomático del impreso incompleto de 1633 que existía en la Biblioteca Nacional de México. Se ha extraviado.]

LÓPEZ DE CoGolludo, Diego

1957 Véase Rubio Mañé, J. Ignacio (1957).

**MSAKeMSON, M. W.

1951 The Book of the Jaguar Priest: A Translation of the Book of the Chilam Balam of Tizimin, with Commentary, Henry Schuman, Nueva York.

**Mediz Bolio, Antonio

1930 Libro del Chilam Balam de Chumayel, traducción del idioma maya al castellano, San José de Costa Rica. 
MORLEY, Sylvanus G.

1949 Véase Barrera Vásquez, Alfredo (1949).

Relaciones

1983 Relaciones histórico-geográficas de la Gobernación de Yucatán, 2 tomos, UNAM, Instituto de Investigaciones Filológicas, Centro de Estudios Mayas, México. (Fuentes para el Estudio de la Cultura Maya, 1.)

RENDÓN, Silvia

1969 Véase Barrera Vásquez, Alfredo (1969).

Ritual

1987 El ritual de los Bacabes, edición de Ramón Arzápalo Marín, UNAM, Instituto de Investigaciones Filológicas, CEM, México. (Fuentes para el Estudio de la Cultura Maya, 5.)

Roys, Ralph Loveland

1949 "The Prophecies for the Maya Tuns or Years in the Books of Chilam Balam of Tizimin an Mani", en Contributions..., 10/51: 155:186.

1967 The Book of Chilam Balam of Chumayel, introduccion de J. Eric S. Thompson, Norman, University of Oklahoma Press. The Civilization of the American Indian Series. [ $1^{2}$ edición en 1933, por la Carnegie Institution of Washington.]

1968 Véase Scholes, France V. (1968)

RuBio MaÑé, J. Ignacio

1957 "Notas y acotaciones" a la Historia de Yucatán, de fray Diego López de Cogolludo, O.F.M., 2 tomos, Editorial Academia Literaria, México. [Reproduce en facsímil la edición madrileña de 1688.]

SAHAGÚN, Bernardo de

1585 "Arte adiuinatoria que vsaban los Mexicanos en tiempo de su ydolatría...", texto contenido en el volumen que se conoce como Cantares megicanos, ms., sin fecha en la Biblioteca Nacional de México, pp. 101-125 [117-142].

Scholes, France V., y Ralph L. Roys

1968 The Maya Chontal Indians of Acalan-Tixchel, ayuda de Eleanor B. Adams y Robert S. Chamberlain, University of Oklahoma Press, Norman. (The Civilization of the American Indian Series.)

Solf́s AlCalá, Ermilo

1949 Códice Pérez, [Texto maya y] traducción libre del maya al español. Guía (1950) por Ralph L. Roys, en traducción española de Gustavo Vega Ibarra, Ediciones de la Liga de Acción Social, Mérida, Yucatán. 
Thompson, J. Eric S.

1960 Maya Hieroplyphic Writing: An Introduction, University of Oklahoma Press, Norman.

1967 Véase Roys, Ralph L. (1967)

TozZER, Alfred M.

**1917 "The Chilam Balam books and the possibility of their translation", en Procedures of the 19th International Congress of Americanist, Washington, 1915: 178-186.

1921 A Maya Grammar, Cambridge, Massachusetts, Harvard University, Papers of the Peabody Museum, vol. IX. [Reedición por Dover Publications, Inc., Nueva York, 1977.]

**Villagutierre Soto-Mayor, Juan de

1701 Historia de la conquista de la provincia de Itzá..., Madrid. [Hay edición hecha en Guatemala, en 1933.] 